\title{
CCIRGTN-Coexistence Channel Interference Reduction Using Game Assumptions in Television White Space Network
}

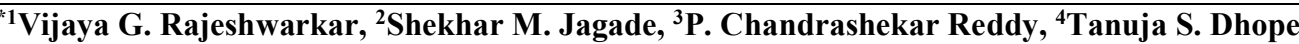 \\ ${ }^{1,3}$ Jawaharlal Technological University Hyderabad, ${ }^{2}$ STB's College of Engg. Tuljapur \\ ${ }^{4}$ BVUCOE, Pune \\ *Email:v.rajeshwarkar@gmail.com
}

Received: $13^{\text {th }}$ December 2018, Accepted: $13^{\text {th }}$ February 2019, Published: $30^{\text {th }}$ June $_{2019}$

\begin{abstract}
In the last decade, wireless communication growth has been created not only because of exclusive openings, but also it formed big challenges to the market. So as to meet the increasing demand at no too much cost or power utilization, one possible solution for the providers is to obtain more channels for wireless transmissions. Unlike the long period channel assignment to restricted licenses, secondary channel admission is considered as an acceptable option to get extra channels at the minimum cost. In specific, the very high-frequency and ultra highfrequency television band is measured as the most capable solution for secondary usage because of its better channel propagation characteristics. This proposed CCIRGTN, Coexistence Channel Interference Reduction using Game assumptions in Television white space Network, discusses the neighboring channel coexistence interference minimization techniques. Based on the interference device detection probability, removal of noninterference devices within the range of interference devices is initiated. After that, network starts the device reconnection based on the position of the devices and its interference range devices. The probability estimation is applied based on the connected devices and time period of collecting the television band devices (TVBD) network information. From the distributed random probability, decision making is executed to initiate the game concept between devices. The game records the reports on the number of players and game plans. Based on the gaming output, collect the average power used channel to access currently.
\end{abstract}

Keywords

Channel Demand; Channel Power; Interference; Coexistence White Space; TVBD Network; Probability Estimation; Gaming

\section{Introduction}

Dynamic channel selection allows channel sharing with available authorized/licensed services such as satellite, radar communications, TV telecasting etc. The aim of channel sharing is that, it finds and eliminates the sub-channel interference along with confined equipment by dynamically choosing a band which is not in use. This method brings channel sharing techniques to choose the most excellent channel, it may be a less interfered or non-interfered channel, as per the frequency capacities.

Among the channel frequencies it is possible to access the very high frequency (VHF) and ultra high frequency (UHF) bands used for digital television (TV) broadcasting tune-up, also named as TV-bands. Basic TV broadcasting environment contains high-power TV transmitters and towers. So as to reduce the internal noisiness or to telecast the regional programs, that is broadcasted through the dissimilar frequencies of all TV towers needs proper solutions. Besides broadcasting, the propagation activities on TV frequency bands are highly favourable for broad region coverage range or internal-outside transmission range than the maximum of the channel bands utilized for mobile phone infrastructures. So that, the plentiful channels in the TV frequencies have paid attention to public and research areas.

This dissimilar set of wireless systems could face the interference problem of its deployed areas with a minimum count of TVWS bands. The calculation of WS bands differs from place to place due to the count of television stations operating in an area. TVWS coexistence issue is highly complex and greatly severe so that a perfect significant system among several unrelated white spaces networks and equipment is needed. We focused on the environment specification and technological challenges connected to the solution. It is essential to judge the states in which the WS bands are being used and recognize the factors producing coexistence issues and the solutions. If dissimilar wireless systems, like an IEEE 802.19.1 and an IEEE 802.11 are placed in a common area there is a possibility for interference. If they work on the similar WS band it is possible for any one of the networks to face interference from the other network.

The unused or underutilized frequencies in the TV band range are known as TV White Spaces or spectrum holes. TV telecast bands have individual attention since new move towards TV band holes for allowing broad area services are being measured and their broadcast uniqueness is in fact good for channel communications. The truth that TV channel bands presents good conditions to wireless networks and the need of additional bands ever-increasing demands, motivated the enhancement of the new inventions. 
A frequency of channel can be measured underused if it can agree to allow the secondary broadcasts without damaging the process of main transmissions. The area of distance, time, frequency in which secondary utilization is possible are named as a frequency hole. In any way if a band is not in use for a specified time, the usage of this band by others can create interference from neighboring band users, so the idea is to give adequate benefit to other users while shielding main clients from interference. So that a band hole is mentioned as a spectrum band in which secondary users can send with no interference to the main users.

\section{IEEE 802.11}

Listen before sending data is a known technique for professionally sharing a channel between several mac802.11 protocols. The concept of $802 \_11$ is that before sending packets over a distribution channel, a transmission device verifies if the shared band is in usage by sending a request to send (RTS) message. If it receives channel clearance message was clear to send (CTS) from one of its neighbouring equipment it can start its transmission. If the equipment does not listen to the CTS within the predefined time threshold, the transmitter shall postpone its deal by a random time so that the sending equipment gives sensible chances to remaining equipments.

\section{IEEE 802.19.1}

The 802.19.1 MAC protocol has few logical objects noted by its efficient roles and their interfaces connected with outside objects. The specific objects are CM, CE and CDIS and the notable interfaces are: Interface A: Interface B1: Interface B2: Interface B3: Interface C: and Interface D as shown in Fig.1. Also it is connected with the other outside objects such as TVWS database, TVBD devices and management entity.



Figure 1: 802.19.1 Internal Connection View [Source 14]

The game concept considered is based on the factors like the player's count, the summation of the achievements or failures, and the way of strategies placed in the game. The expressions used in the game are channel selection, by varying multiple conditions used to select the channel.

This paper designed as follows: Section 2 presents the related works of the discussion of the previous papers. Section 3 presents the performance analysis of the CCIRGTN along with the different MAC support. Section 4 proposes a CCIRGTN based finest channel selection using the game model in TVWS network. Results are discussed in Section 5. Section 6 concludes the paper.

\section{Related Work}

The decision makers in the game are rational users or network operators who control their communication devices. These devices have to cope with a limited transmission resource that imposes a conflict of interests [3]. One alternative mode of spectrum utilization that is finding popularity world over is the use of TV White Spaces for communications systems [1]. A large amount of spectrum is being made available with the migration from the analogue to digital transmission throughout the country through effective planning. Digital Dividend refers to the 'leftover frequencies' that results from the change of broadcasting from analogue to digital domain [5]. The White-Fi, a UHF white space wireless network that adaptively configures itself to operate in the most efficient part of the available white spaces [2]. The air interfaces must be standardized to reduce price of wireless unit and to increase the number of unit suppliers, and to expand application Fields [6]. TV white space depends on regulations such as the protection margin to the primary user, maximum height above average terrain (HAAT), transmission power of secondary user, and the separation distance [4]. This has led to the development of different techniques for addressing the spectrum crunch and also to optimize the use of the available spectrum. The TV White spaces have excellent propagation characteristics and hence are a huge attraction worldwide for wireless communications [11]. WSDs obtain the available spectrum information through querying the geo-location database, instead of performing spectrum sensing [8]. Standards and interoperability across protocols and networks are often 
poorly specified, and in general, "cellular" reflects a complex ecosystem, tightly controlled by providers and hardware vendors [10]. This potentially makes them suitable for use in rural broadband applications, where transmission links may be several kilometres in length and may involve challenging terrain such as hills, foliage, and water [7]. The applications of different game theoretic frameworks to model wireless communications problems relating to directional antennas, dynamic spectrum access, infrastructure sharing, cloud-assisted radio access, cooperative content delivery[12]. Wi-Fi and these other devices contend for the same channel-constrained $2.4 \mathrm{GHz}$ frequency spaces making it more crowded and unsuitable for Wi-Fi use due to too much interference from other devices and the Wi-Fi connections [9].

\section{CCIRGTN - Network Design and Functions}

The process of TV white space sequence is beginning with the association of the TV band device network TVB. Network is deployed with the set of internet server (IS), Data base server (DBS), the coexistence discovery and information server CDIS server, coexistence manager (CM), coexistence enabler (CE), access point (AP), TV station broadcasting and set of clients (C).



Figure 2: TVBD Network Connection

It operates the television broadcast spectrum at the specific locations where it is allowed. CE can be deployed inside the TV band device or network. CDIS can then be deployed outside of TV band networks.

\section{A. Efficient Role of Coexistence Enabler (CE)}

The Coexistence Enabler CE is responsible for the communication between the CM and a TVBD network. Coexistence information is obtained by CE from a TVBD network or a device, and it interprets the reconnecting queries or instructions and control specifications received from the CM into TVBD specific reconnecting queries or instructions. TVBD network designing is initiated into the process of finding the located devices inside the coverage region of the TVBD network.

\section{B. Efficient Role of Coexistence Manager (CM)}

$\mathrm{CM}$ is responsible to find the other CMs and creating coexistence-connected verdicts in order to resolve the coexistence issues raised by the TVBD networks what they are serving up. They also provide coexistence instructions and control specifications to CEs and support network providers to deal with the coexistence problems.

$\mathrm{CM}$ can provide the support to take decisions on the peer networks and hierarchical networks. It can easily connect with the interfaces between other CMs. Also it can collect and combine the particulars about coexistence issues easily. Additionally CM contains the processing and storage capabilities.

Therefore coexistence issues detected devices are striking as whitespace (WS) devices. For each WS device, the interference range devices are detected and needed alternate the network service allotment is performed by the TVBD network.

\section{Efficient Role of CDIS}

The CDIS provides the coexistence information to CMs, and Interfaces between CMs. CDIS also gathers and combines the information related to TVWS coexistence, and may join the TVWS database to obtain the information on current user channel utilization [13].

\section{Neighbor Detection Process (NDP)}

The NDP is run inside the CDIS to decide the possibility of harmful interference in two TVWS networks. The input parameters of the NDP are:

- Well Known positions of the main TVBD and number of connection devices in the network

- Network atmosphere level, interior, exterior, rural, urban areas, etc.

- Antenna height - Transmission power and receiving power of antennas, directivity of antenna and sensitivity

- Operating bands and channel bandwidth

\section{E. Interference Observations}

- $\quad$ Find each network's channel demand based on the amount of channels requested by a network.

- Conclude the interference level graph (ILG) which shows the connections of interference between the coexisting devices and networks. 
ILG is a graph that it explains the devices are representing the TVBD networks and their borders join the networks that interfere with each one. The weight of a border denotes the smallest amount of the band isolation to get a threshold level of signal to noise ratio of any one of the networks connected by the border among the two interfering networks.

\section{F. Propagation Based Interference}

The protocol chooses the signal propagation as per the network environment nature and computes the interference range of the networks that minimizes the joint-channel and neighbouring connected channel interference. To bring out this mission, the protocol uses an ILG that gives the certain measured specification on the neighbouring-channel and joint-channel interference from each set of networks within the interference region of each one. The device ILG is resolute based on a position, power of transmission and receiving, channel behaviour, and available band. Also, it uses this ILG to know the least level of channel band isolation from interfering range networks and update this value into the ILG.

Also, it creates the maximum ILG based channel propagation based on the possible interfering links between two connected networks and evaluates possible interference between networks in all directions.

\section{G. Interference Device Probability}

According to the interference range device probability, update the information to remove the non coexistence devices from the interference range devices. The conclusion is designed for CM to take the working decision based on the parameters. CM keeps a list of each device in TVWS network and it contains the complete information about the network also. Using the list of information CM creates a precedence list of networks. Uppermost level precedence is fixed to 1, that networks may not have a channel to connect or have a strange interferer. In addition, device with peak signal error rate and minimum participation is added to the precedence list. According to this, the CM verifies the chances to reconnect the chosen TVWS networks. If not, the CM checks the next alternative solutions that need reconnection of neighbours.

\section{H. Device Reconnection}

After achieving the information update, the device reconnection is executed based on the position of the normal devices and its interference range devices placed inside the range. The count of WS channels, which differs from place to place, depends on the volume of TV stations in service in the selected area. The number of channels reduced still because of the other equipment used. So that, the WS channel usage outline can differ from each day or time to time. If dissimilar networks, like an 802.19 and an 802.11 are placed in a frequent area there is a possibility for interference. The evaluation step is built up from the probability of transmission based on the attached devices and time duration of gathering the TVBD network information.

\section{Channel Interference Decision based on Gaming Concept}

From the distributed random probability, decision making is initiated into the gaming verdict. Through this judgment, measure the consistency of the verdicts on the outcomes and viewpoint about the possibility of these outcomes. The game results are built up with the n-number of player's and game plans, with the gaming restrictions together with the channel payoff matrix and stability matrix. The WS device collections of the TVBD network are the representation of the players for more than one player game. The basic decision step describes the game that contains the collection of players, a group of perfect plans for each game player, and a detail list of each game player, and the game player's payoff roles.

The game plan and collection of player's payoff are equally started by the null payoff game matrix. Sequentially to fill up the payoff game matrix of a player, utmost channel band of the game is validated from the channel list containing the player's and plans. A model game list can be signified by rows (r) and columns (c) in matrices related to the players (PL), plans (PN), and the values of the payoffs from every player. The enclosed Table 1 represents the possible game values of channel interference power (CIP), symmetric channel (SC) values and the asymmetric channel (AC) values.

\begin{tabular}{|l|l|l|l|}
\hline & c1-PN-SC & c2-PN-AC & C3-PN-CIP \\
\hline R1-PL1 & 0.000104 & 0.836493 & $8.66 \mathrm{E}-05$ \\
\hline R2-PL2 & 0.000426 & 0.214862 & $9.15 \mathrm{E}-05$ \\
\hline R3-PL3 & 0.000101 & 0.688981 & $7.81 \mathrm{E}-05$ \\
\hline R4-PL4 & 0.000101 & 0.830771 & $8.41 \mathrm{E}-05$ \\
\hline
\end{tabular}

Table 1: Payoff Matrix

Where, PL1,PL2,PL3, and PL4 describes the different players as shown in Table 1. The matrix shows the samples of rows and columns, which contains the perfect plans and relevant players: columns and rows respectively. By using these reports possible plan based summary can be created, each one linked with an outcome applicable to the players based on the CIP, SC, and AC.

A perfect plan defines the possibility of present activities in games in a planning form. The planning concept includes the maximum possibilities in widespread games and varied planning as per the CIP. Here the symmetric channel SC denotes the maximum equivalent values and the asymmetric channel value explains the deviations. 
From the values of the CIP of each player and the maximum power value finds from the payoff table matrices, then the equilibrium matrix is filled with the normalized power value required for accessing the spectrum band.

In broad games, a plan specifies an activity for each device in the game hierarchy at which a player has to take next move. Here, the PL1 is using a channel with maximum power. When nearby device wants to send a transmission it can choose L or R. If PL2 decides to communicate, without knowing which channel player 1 is working, this payoff matrices brings the solution to take the channel without interfering, the model Figure drawn in Fig. 3.

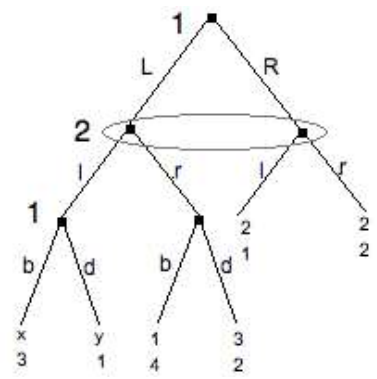

Figure 3: Game Hierarchy

In addition, the power needed for the payoff is selected from the equilibrium matrix. Now the game is initiated between each player by comparing the CIP of each other players. So that, the game uses a Nash equilibrium (NE) solution, it brings the better adaptability conclusions. The NE applies to the planning summary (PS) as needed. The CIP is computed from the total power of the maximum absolute difference of the payoff power value of each other player. The NE table is shown in Table 2.

\begin{tabular}{|r|r|r|r|l|r|}
\hline 1 & 0.243087 & 0.991269 & 0.946376 & NEQULI & 0.72691 \\
\hline 2 & 1 & 0.254618 & 1 & NEQULI & 0.751539 \\
\hline 3 & 0.266246 & 0.816462 & 0.853748 & NEQULI & 0.645486 \\
\hline 4 & 0.237878 & 0.984488 & 0.919762 & NEQULI & 0.714043 \\
\hline
\end{tabular}

Table 2: NE Matrix

Now the payoff plan for each one of the player is created as the percentage of current CIP of the device and maximum CIP among the all devices. The varied NE seems uneven. If the column knew which perfect player means row would start its play. Based on the two or more potential outcomes, the NE loses maximum of its demands. The NE is played with its techniques that direct all the participated players to wait for the similar equilibrium.

Channel payoff matrix is formed for Interference, Symmetric and Asymmetric is to know the interference power, total interference and payoff of the user respectively. Among all the players payoff, maximum strategy for SC, AC and CIPs are computed for performing the NASH Equilibrium.

The state of equilibrium for each channel payoff is computed as per the sum of each state. The final NE for the power allocation is computed as per the average power required with the n-player gaming strategy.

\section{Results and Discussion}

To confirm the suitable operation of the implementation, few tests were carried out. This discussion shows the results of various scenarios and its validations. The network delay, throughput, used overhead counts and received packet delivery ratio outputs are presented here by varying the network traffic generation time as the interval.

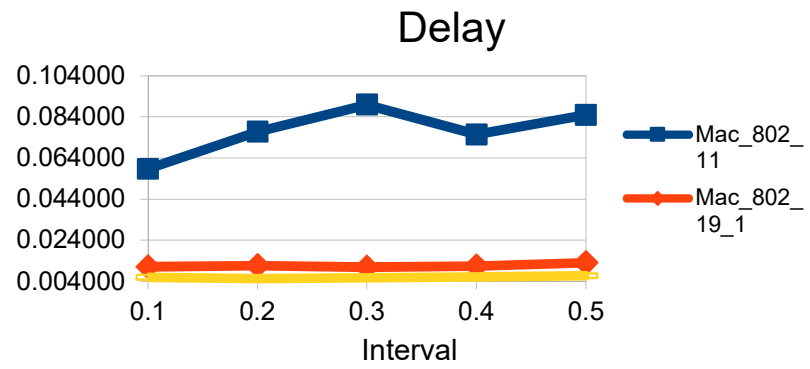

Figure 4: Packet Interval Vs Delay 
This delay graph of fig. 4 shows the time duration to communicate with one device to end of the device. Due to the interference reduction in a network using the game, each device gets the minimum interfered or no interference channel. Based on the minimum average power requirement channel selection minimizes the delay in fig.4. CCIRGTN built with the MAC_802_19.1 Mac_802_11 modified with super-Fi technology and checked to compare with the $802 \_19.1$.

\section{Control Overheads}

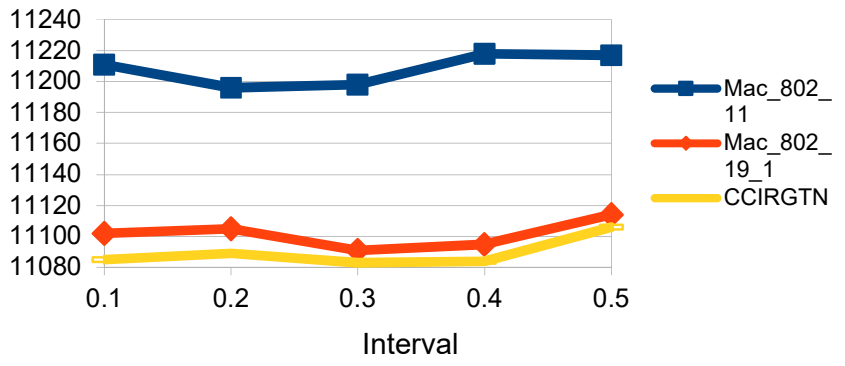

Figure 5: Interval Vs Overhead

The control overheads considered from the network establishment packets, if the interference is less, also if the game selects the proper channel to communicate the network; it returns the lowest control packets. In Fig.5 CCIRGTN with Mac_802_19.1 shows the least control packet usage because of the optimized channel selection using the game and interference reduction. Minimum control packets improve the channel utilization time period.

\section{Packet Delivery Ratio}

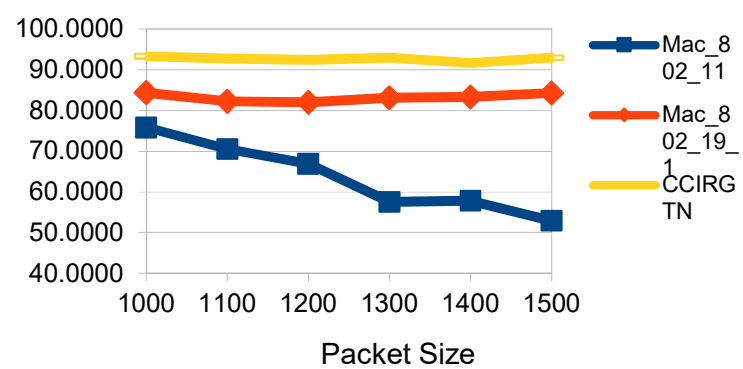

Figure 6: Packet Delivery Ratio

Fig. 6 shows the packet delivery ratio of CCIRGTN compared with the coexistence autonomous decisionmaking protocol for white space of CPDA. Here the CCIRGTN shows the maximum packet delivery ratio output than the other comparisons. Because of the internal working technology of CCIRGTN, due to the delay reduction and minimum control overhead receiving packets increased. Between the devices, the signals are transmitted with proper channel selection.

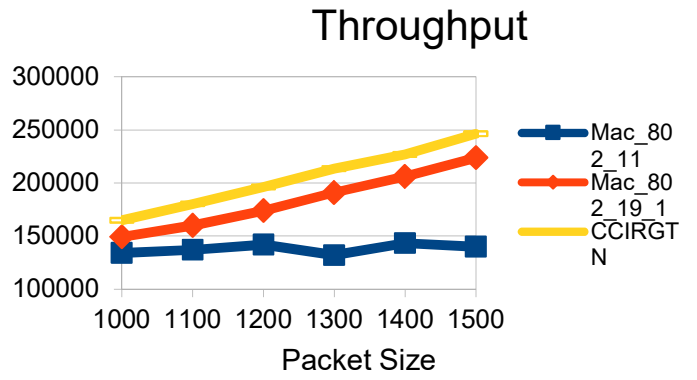

Figure 7: Throughput

The bits per second receiving count considered as throughput. Here in Fig.7 the output shows the throughput results from CCIRGTN and CPDA of existing. If the receiving packet counts increased from the packet delivery ratio, the receiving bits per second also increased. It shows stable network communication based on the designed protocol. 


\section{Conclusion}

CCIRGTN- Coexistence Channel interference Reduction using Game assumptions in Television white space Network system works well as compared to MAC 802.11. Simulation results show that this system shows results approximately in accordance with MAC 802.19.1. The game model ends in Nash Equilibrium. It can thus be concluded that this is a better interference reduction technique

\section{References}

1.Dimitris E. Charilas, Athanasios D. Panagopoulos "A survey on game theory applications in wireless networks" Computer Networks, 17 July 2010.

2. Radio Division, "Broadband deployment through TV-White Space" Telecommunication Engineering Center, 2008.

3. Jai Sachith Paul, Akhil P Sivan, Kumar Raushan Ratnesh, Sreekala K "Effective Deployment of TV White Space for Enhancing Rural Broadband in India", International Journal of Computer Science and Mobile Computing, ICMIC13, December- 2013.

4. Paramvir Bahl, Ranveer Chandra, Thomas Moscibroda, Rohan Murty, Matt Welsh "White Space Networking with Wi-Fi like Connectivity”, Aug 2009.

5. Hiroshi Harada "White Space Communication Systems: An Overview of Regulation, Standardization and Trial", Institute of Electronics, Information and Communication Engineers, IEICE TRANS. COMMUN., VOL.E97-B, NO.2 FEBRUARY 2014.

6. Gaurang Naik, Sudesh Singhal, Animesh Kumar, and Abhay Karandikar, "Quantitative Assessment of TV White Space in India“, Oct. 2013.

7. Manjurul H. Khan, P.C. Barman, “TV White Space in Rural Broadband Connectivity in Case of Bangladesh toward "Vision 2021", American Journal of Engineering Research (AJER),Volume-7, Issue-3, 2018.

8. Yuan Luo, Lin Gao, and Jianwei Huang, "Spectrum Reservation Contract Design in TV White Space Networks", 2 Nov 2015.

9. Ghufran Baig, Dan Alistarh, Thomas Karagiannis, “Towards unlicensed cellular networks in TV white spaces", Association for Computing Machinery, December 2017.

10. Miss. Shruti S. Kshirsagar, Prof. Amit G. Fulsunge "A Review: Analysis of White Space for Designing Communication Module", International Journal on Recent and Innovation Trends in Computing and Communication (IJRITCC), Volume: 2 Issue: 11,November 2014.

11. Zaheer Khan, “Game Theory for Wireless Communication Networks”, Centre for Wireless Communications (CWC), University of Oulu,2018.

12. Hope Mauwa, Antoine Bagula, \& Marco Zennaro, "Exploring TV White Spaces for use in Campus Networks", ICST Institute for Computer Sciences, Social Informatics \& Telecommunications Engineering, 2016.

13. Tuncer Baykas, Mark Cummings, et.al. "Developing A Standard for TV White Space Coexistence: Technical Challenges and Solution Approaches", in IEEE Wireless Communications, Vol.19, Issue 1, Feb.2012, PP-10-22.

14. Duzhong Zhang, Quan Liu, Lin Chen and Wenjun Xu, "Survey on coexistence of heterogeneous wireless networks in $2.4 \mathrm{GHz}$ and TV white spaces", International Journal of Distributed Sensor Networks, Vol. 13(4), 2017. 